\title{
Esquemas maladaptativos tempranos y trastornos de ansiedad en la primera infancia y la niñez
}

\author{
Early Maladaptive Schemas and anxiety disorder in infancy \\ and childhood
}

\begin{abstract}
Primeiros esquemas mal adaptativos e transtornos de ansiedade na infância
\end{abstract}

\author{
José Luis Álvarez Posada** \\ Antonio José Villa Londoño*** \\ Recibido 28. 02. 2016 • Arbitrado 26. 03. 2016 • Aprobado 13. 04. 2016
}

\section{Resumen}

El artículo presenta resultados de una investigación orientada a identificar la presencia de esquemas maladaptativos tempranos y trastornos de ansiedad en niños de 2 a 6 años de edad de la ciudad de Medellín, y determinar si existe una correlación estadísticamente significativa entre esas dos variables. El estudio realizado fue de tipo cuantitativo, de nivel correlacional y de enfoque empírico analítico. La muestra de participantes fue por conveniencia, y se emplearon como instrumentos y técnicas de recolección de información el cuestionario de esquemas maladaptativos tempranos para niños, y el cuestionario para la identificación de cuatro trastornos de ansiedad (ansiedad ge-

* Investigación realizada en la Universidad de San Buenaventura, Medellín, en 2015

** Psicólogo, Universidad de San Buenaventura, Medellín; doctor en neurociencias de la Universidad de Maimónides, Argentina; docente tiempo completo Universidad de San Buenaventura, Medellín, Colombia, jlap.cat@gmail.com

*** Psicólogo, Universidad de San Buenaventura, Medellín; especialista, Universidad de San Buenaventura, Medellín; Magister, Universidad de San Buenaventura, Medellín; doctor en Neurociencias de la Universidad de Maimónides, Argentina; docente tiempo completo Universidad de San Buenaventura, Medellín, Colombia, anjovi59@gmail.com 
neralizada, estrés postrauma, fobia específica y trastornos de pánico), ambos diseñados para la investigación. El estudio brinda datos importantes sobre la relación entre los EMT y los trastornos de ansiedad, información de utilidad para los psicólogos clínicos. La investigación está enmarcada dentro de la resolución número o08430 de 1993 (4 de octubre de 1993), por la cual se establecen las normas científicas, técnicas y administrativas para la investigación en salud.

Palabras clave: esquemas maladaptativos tempranos, trastornos de ansiedad, infancia, teoría del apego.

\section{Abstract}

The article presents results of a research to identify the presence of early maladaptive schemes and anxiety disorders in children 2-6 years old in the city of Medellín, and determine whether there is a statistically significant correlation between these two variables. The study was quantitative, correlational level and empirical analytical approach. The participant sample was for convenience, and used as tools and techniques for information gathering: a questionnaire of early maladaptive schemes and a questionnaire for the identification of four anxiety disorders (generalized anxiety, post-traumatic stress, specific phobia and panic disorder), for children, both designed for research. The study provides important data on the relationship between EMT and anxiety disorders, useful information for clinical psychologists. The research is framed within resolution number 0084301993 (4 october 1993), by which are established the standards scientific, techniques and management for health research.

Keywords: early maladaptive schemas, anxiety disorders, infancy, childhood. 


\section{Resumo}

$\mathrm{O}$ artigo apresenta resultados de uma estratégia para identificar a presença de esquemas maladative precoces e transtornos de ansiedade em crianças de 2 a 6 anos de idade, da cidade de Medellín, e determinar se existe uma correlação estatisticamente significativa entre estas duas variáveis de pesquisa. O estudo foi quantitativo, o nível de correlação abordagem analítica e empírica. A amostra de participantes foi por conveniência, e utilizados como ferramentas e técnicas para coleta de informações ele questionário esquemas maladative da primeira infância e questionário para a identificação de quatro transtornos de ansiedade (transtorno de ansiedade generalizada, estresse pós-traumático, fobia específica e distúrbios pânico), ambos concebidos para a investigação. $O$ estudo fornece dados importantes sobre a relação entre EMT e transtornos de ansiedade, informação útil para os psicólogos clínicos. A pesquisa está enquadrada dentro da resolução numero 008430, de 1993 (4 de outubro de 1993), pelo qual as informações científicas, técnicas e administrativas para os padrões de pesquisa em saúde estão estabelecidas.

Palavras-chave: primeiros regimes de má adaptação, transtornos de ansiedade, a teoria do apego infantil.

\section{Introducción}

La terapia centrada en esquemas de Jeffrey Young fue creada inicialmente para optimizar la tradicional terapia cognitiva de Beck, integrando elementos de la teoría del apego de John Bowlby para profundizar en la comprensión del desarrollo emocional en los niños, al igual que aportes de la terapia procesal sistémica de Vittorio Guidano quien resalta igualmente la importancia del papel que juegan los primeros años de vida y la relación con los cuidadores en el desarrollo del psiquismo personal. El modelo focalizado en esquemas contiene varias modificaciones importantes al trabajo original de Beck (Caro, 1997)

Retomando varios elementos, Young desarrolló su conceptualización de los esquemas maladaptativos tempranos, con un alto conte- 
nido emocional y de origen pre verbal que se crean desde el inicio del vínculo del niño con su cuidador.

A lo largo de los años, dicho modelo ha logrado consolidarse como una de las propuestas más completas e integradoras de la escuela cognitiva a nivel internacional.

Por otro lado, las tradicionales explicaciones de la etiología de los trastornos psicológicos han tenido en cuenta conceptos como el de los supuestos subyacentes de Aarón Beck y las ideas irracionales de Albert Ellis, pero se hace necesario indagar más a fondo sobre el rol que juegan los esquemas maladaptativos tempranos en la génesis de la psicopatología. Existen estudios sobre los miedos en la infancia, como el de los miedos en la infancia y la adolescencia: un estudio descriptivo (Méndez, 2003), pero estos no retoman la variable de los esquemas maladaptativos tempranos.

En esta investigación se hace una aproximación desde la teoría de los esquemas maladaptativos tempranos para ampliar la comprensión con respecto a la relación entre los EMT's y la psicopatología, específicamente de cuatro trastornos de ansiedad, relación que según los resultados obtenidos parece fundamental desde los primeros años de vida, pues se logra, a partir de los datos obtenidos y su posterior análisis, determinar que sí existe una relación significativa entre dichos esquemas y los trastornos abordados en el grupo de niños evaluados.

En el análisis de resultados, además de mostrar dichas relaciones, se hace una aproximación explicativa de las mismas, esto para que sea de utilidad en los contextos de la psicología clínica en donde se abordan las variables aquí investigadas.

\section{Referente Teórico}

\section{Esquemas maladaptativos tempranos}

Los EMT's son el resultado de experiencias disfuncionales recurrentes, están estrechamente ligados a altos niveles de afecto y sensaciones corporales, constituyen una abstracción fruto de la experiencia 
previa, son organizaciones de significados personales referentes al sí mismo, los eventos, las personas y las cosas. Se desarrollan a partir de la relación del niño con su cuidador (Young, 1999).

\section{Areas de desarrollo y esquemas maladaptativos tempranos}

Área de la autonomía: esta área hace referencia a la capacidad del sujeto para funcionar independientemente en el mundo, sin el continuo apoyo de los demás. Los individuos autónomos son capaces de expresar sus necesidades, intereses, preferencias, opiniones y sentimientos (Young, 1999). Los EMT's de esta área son: dependencia/ incompetencia, subyugación/sumisión perdida de la individualidad, vulnerabilidad al daño y a la enfermedad y temor a perder el autocontrol/inhibición emocional.

Área de las relaciones interpersonales: esta área hace referencia a la sensación de que el sujeto está conectado (relacionado) con otras personas de manera estable, duradera y confiable. Una primera forma de conexión se refiere a la intimidad (Young, 1999). Los EMT's de esta área son deprivación emocional/desamor, abandono/pérdida, desconfianza/abuso y aislamiento social/alejamiento.

Área de la autovaloración: esta área se refiere al desarrollo de la sensación de ser digno de ser amado, competente, aceptable y deseable para los demás y de ser digno de su atención, amor y respeto (Young, 1999). Los EMT's de esta área son: defectuosidad/inamabilidad, indeseabilidad social, incompetencia/falla, culpa/castigo y pena/vergüenza.

Área de límites razonables: se refiere a la capacidad de disciplinarse uno mismo, controlar sus impulsos y tener en cuenta las necesidades de los otros, todo en un grado apropiado (Young, 1999). El EMT's de esta área es: límites insuficientes/engrandecimiento.

Área de los estándares: el área de expectativas razonables tiene que ver con la capacidad de determinar de forma realista, estándares alcanzables tanto con respecto a uno mismo como a los demás, especialmente en cuanto toca o se refiere a los desempeños y los logros (Young, 1999). El EMT’s de esta área es: estándares inflexibles/hipercrítica. 


\section{Procesos de mantenimiento de esquemas}

Se refiere a los procesos mediante los cuales se refuerzan los esquemas maladaptativos tempranos (EMT's). Estos procesos incluyen tanto estilos de pensamiento específicos poco viables, como patrones conductuales de auto derrota relacionados con las profecías autor realizadas (se generan comportamientos que al influir sobre el medio ambiente terminan retroalimentando y confirmando el esquema). También incluyen la rigidez y la dificultad para considerar otras reglas internas de funcionamiento $\mathrm{u}$ otras formas de ver el mundo, generando una gran resistencia al cambio (Young, 1999).

\section{Los diferentes tipos de mantenimiento esquemático son:}

Mantenimiento o rendición: sobrevaloración o magnificación de la información que los confirma, comportamientos de auto derrota; evitación: intentos automáticos o voluntarios por bloquear pensamientos o imágenes que puedan activar el esquema, despersonalización, evitación afectiva, evitación de situaciones de la vida real; sobrecompensación o contra ataque: el sujeto termina comportándose de manera totalmente contraria a lo que se esperaría teniendo en cuenta su EMT's (Young, 1999)

\section{Trastornos de Ansiedad}

Existen unos criterios universales para determinar si el comportamiento de una persona cabe diagnosticarlo como un trastorno de ansiedad. Estos criterios están recogidos en las dos clasificaciones de trastornos mentales más importantes: DSM-V (Asociación de Psiquiatras Americanos, 2014) y ICD-10 (Organización Mundial de la Salud, 2016).

Los trastornos de ansiedad no se diferencian entre sí mucho por las manifestaciones o respuestas de ansiedad, sino que más bien se encuentran diferencias entre dichos trastornos ligadas a las áreas situacionales en las que los sujetos con diferentes trastornos de ansiedad presentan dificultades o altos niveles de ansiedad (SEAS, s.f.). 
Pánico: trastorno de ansiedad que se caracteriza por la aparición temporal y aislada de miedo o malestar intensos, acompañada de una variedad de síntomas fisiológicos, cognitivos, emocionales y conductuales, que se inician bruscamente y alcanzan su máxima expresión en los primeros 10 min (DSM-V, 2014).

Fobia Específica: Temor acusado y persistente que es excesivo o irracional, desencadenado por la presencia o anticipación de un objeto o situación específicos (p. ej., volar, precipicios, animales, administración de inyecciones, visión de sangre) (DSM-V, 2014).

Estrés Postrauma: trastorno de ansiedad que se caracteriza por recuerdos del acontecimiento recurrentes e intrusos que provocan malestar y en los que se incluyen imágenes, pensamientos o percepciones, sueños de carácter recurrente sobre el acontecimiento, que producen malestar, la sensación de que el acontecimiento traumático está ocurriendo, malestar psicológico intenso al exponerse a estímulos internos o externos que simbolizan o recuerdan un aspecto del acontecimiento traumático y evitación persistente de estímulos asociados al trauma, todo esto dentro de un contexto donde la persona ha sido expuesta a un acontecimiento traumático tales como muertes o amenazas para su integridad física o la de los demás y ha respondido con un temor, una desesperanza o un horror intensos. En los niños estas respuestas pueden expresarse en comportamientos desestructurados o agitados (DSM-V, 2014).

Ansiedad Generalizada: trastorno de ansiedad que se caracteriza por ansiedad y preocupación excesivas (expectación aprensiva) y una cariada gama de síntomas fisiológicos, cognitivos, emocionales y conductuales, sobre una amplia gama de acontecimientos o actividades (como el rendimiento laboral o escolar), que se prolongan más de 6 meses y que al sujeto le resulta difícil controlar (DSM-V, 2014). 


\section{Metodología}

Tipo de estudio cuantitativo; nivel correlacional y enfoque empírico analítico.

Alcance: es una investigación estratégico prospectiva, siendo ésta un estudio que está dirigido a la generación de planes de prevención clínica de análisis de escenarios.

Instrumentos y técnicas de recolección de información: cuestionario de esquemas maladaptativos tempranos para niños de 2 a 6 años de edad y Cuestionario de cuatro trastornos de ansiedad para niños de 2 a 6 años de edad. Ambos cuestionarios fueron diseñados para la investigación y validados bajo juicio de expertos.

Población: conformada por 70 niños entre 2 y 6 años de edad, pertenecientes al jardín infantil Coqui de Medellín.

Muestra: 70 niños (margen de error $=5$; heterogeneidad $=50 \%$; nivel de confianza $=95 \%$ ).

Criterios de inclusión: Niños con edades de 2 a 6 años pertenecientes al jardín infantil Coqui.

Criterios de exclusión: niños con historia previa de discapacidad cognitiva y/o que presenten características del espectro autista.

Variables: esquemas maladaptativos tempranos (áreas de autonomía, relaciones interpersonales, autovaloración, límites y estándares) y cuatro trastornos de ansiedad (ansiedad generalizada, estrés postrauma, fobia especifica pánico)

Hipótesis: Hipótesis Nula $\left(\mathrm{H}_{\mathrm{o}}\right)$ "la relación entre las dos variables de datos no es estadísticamente significativa"; hipótesis alternativa $\left(\mathrm{H}_{1}\right)$, "la relación entre las dos variables de datos si es estadísticamente significativa"

Procedimiento: La información fue obtenida a partir de la aplicación de los cuestionarios directamente al cuidador de cada niño, luego de obtener los datos se procedió a realizar un análisis estadístico a partir del software SPSS 22 determinando el nivel de asociación entre las 
variables a través del chi cuadrado, y posteriormente se continuó con el análisis de los resultados.

\section{Resultados}

La siguiente información fue obtenida a partir del análisis detallado de cada uno de los esquemas maladaptativos tempranos y los trastornos de ansiedad correlacionados.

A partir de los objetivos propuestos para la investigación se pudieron identificar los EMT's de cada uno de los niños participantes en la investigación, igualmente de aquellos que presentaban rasgos clínicos suficientes para determinar la presencia de uno o más de los cuatro trastornos de ansiedad evaluados.

Teniendo en cuenta que las variables son de tipo cualitativo (presencia o no del EMT's , presencia o no del trastorno de ansiedad), para establecer el nivel de asociación, este se calculó con un chi cuadrado (nivel de significancia $=<0.05$ ).

Pertenecen a esta categoría todas aquellas variables sobre las cuales se hace necesario ejercer un control o manipulación con el fin de que éstas no confundan los resultados obtenidos en el grupo con respecto a las variables a observar. Criterio de edad: de 2 a 6; y el sexo. 
Tabla 1. Medidas de tendencia central de las variables demográficas de los 70 participantes de la muestra.

\begin{tabular}{|c|c|c|c|c|}
\hline \multicolumn{5}{|c|}{ Sexo } \\
\hline \multicolumn{3}{|r|}{ Frecuencia } & \multicolumn{2}{|r|}{$\%$} \\
\hline \multicolumn{2}{|c|}{ Masculino } & \multicolumn{2}{|l|}{35} & 50 \\
\hline \multicolumn{2}{|c|}{ Femenino } & \multicolumn{2}{|l|}{35} & 50 \\
\hline \multicolumn{2}{|l|}{ Total } & \multicolumn{2}{|l|}{70} & 100 \\
\hline \multicolumn{5}{|c|}{ Edad } \\
\hline & Frecuencia & $\%$ & media & DS \\
\hline 2 & 11 & 15,7 & \multirow{5}{*}{3,9} & \multirow{5}{*}{1,287} \\
\hline 3 & 18 & 25,7 & & \\
\hline 4 & 18 & 25,7 & & \\
\hline 5 & 13 & 18,6 & & \\
\hline 6 & 10 & 14,3 & & \\
\hline
\end{tabular}

Total $\quad 70 \quad 100$

DS = Desviación Estándar

\begin{tabular}{|c|c|c|c|c|}
\hline \multicolumn{5}{|c|}{ Estrato Socioeconómico } \\
\cline { 1 - 3 } Estrato & Frecuencia & $\%$ & media & DS \\
\cline { 1 - 3 } 2 & 14 & 20,0 & & \\
\cline { 1 - 3 } 3 & 42 & 60,0 & \multirow{2}{*}{3,04} & \multirow{2}{*}{0,731} \\
\cline { 1 - 3 } 4 & 11 & 15,7 & & \\
\cline { 1 - 3 } 5 & 3 & 4,3 & & \\
\hline Total & 70 & 100,0 & & \\
\hline
\end{tabular}

DS = Desviación Estándar

Fuente: Autores

La caracterización de la muestra identifica un grupo de personas con una edad media de 3.9 años, con una desviación estándar de 1.287 años; estrato socioeconómico con una media de 3.04 y una desviación estándar de 0.731. 
Tabla 2. Tabla de contingencia EMT's \& Ttno de Ansiedad Generalizada

\begin{tabular}{|c|c|c|c|c|c|c|c|}
\hline \multirow{3}{*}{$\begin{array}{c}\text { ESQUEMAS } \\
\text { MALADAPTATIVOS TEMPRANOS }\end{array}$} & \multicolumn{7}{|c|}{$\begin{array}{l}\text { Ttno. De Ansiedad } \\
\text { Generalizada }\end{array}$} \\
\hline & \multirow[t]{2}{*}{ Pte. } & \multirow[t]{2}{*}{$\mathrm{f}$} & \multicolumn{2}{|c|}{$\mathrm{Si}$} & \multicolumn{2}{|c|}{ No } & \multirow[b]{2}{*}{$\mathrm{p}$} \\
\hline & & & $\mathrm{n}$ & $\%$ & $\mathrm{~N}$ & $\%$ & \\
\hline \multirow{2}{*}{ Dependencia / Incompetencia } & $\mathrm{Si}$ & $\mathrm{n}$ & 2 & 16,7 & 10 & 83,3 & \multirow[b]{2}{*}{0,531} \\
\hline & No & $\mathrm{n}$ & 6 & 10,3 & 52 & 89,7 & \\
\hline \multirow{2}{*}{ Vulnerabilidad al Daño y Enfermedad } & $\mathrm{Si}$ & $\mathrm{n}$ & 1 & 25,0 & 3 & 75,0 & \multirow{2}{*}{0,380} \\
\hline & No & $\mathrm{n}$ & 7 & 10,6 & 59 & 89,4 & \\
\hline \multirow{2}{*}{ Sumisión / Perdida Individualidad } & $\mathrm{Si}$ & $\mathrm{n}$ & 2 & 40,0 & 3 & 60,0 & \multirow{2}{*}{0,037} \\
\hline & No & $\mathrm{n}$ & 6 & 9,2 & 59 & 90,8 & \\
\hline \multirow{2}{*}{$\begin{array}{l}\text { Temor a Perder el Control / Inhibición } \\
\text { Emocional }\end{array}$} & $\mathrm{Si}$ & $\mathrm{n}$ & 1 & 25,0 & 3 & 75,0 & \multirow[b]{2}{*}{0,380} \\
\hline & No & $\mathrm{n}$ & 7 & 10,6 & $\begin{array}{c}3 \\
59\end{array}$ & 89,4 & \\
\hline \multirow{2}{*}{ Deprivación Emocional / Desamor } & $\mathrm{Si}$ & $\mathrm{n}$ & 2 & 18,2 & 9 & 81,8 & \multirow[b]{2}{*}{0,443} \\
\hline & No & $\mathrm{n}$ & 6 & 10,2 & 53 & 89,8 & \\
\hline \multirow{2}{*}{ Abandono / Perdida } & $\mathrm{Si}$ & $\mathrm{n}$ & o & 0,0 & 10 & 100,0 & \multirow{2}{*}{0,220} \\
\hline & No & $\mathrm{n}$ & 8 & 13,3 & 52 & 86,7 & \\
\hline \multirow{2}{*}{ Desconfianza / Abuso } & $\mathrm{Si}$ & $\mathrm{n}$ & 3 & 23,1 & 10 & 76,9 & \multirow[b]{2}{*}{0,144} \\
\hline & No & $\mathrm{n}$ & 5 & 8,8 & 52 & 91,2 & \\
\hline \multirow{2}{*}{ Aislamiento Social / Alejamiento } & $\mathrm{Si}$ & $\mathrm{n}$ & 3 & 25,0 & 9 & 75,0 & \multirow{2}{*}{0,105} \\
\hline & No & $\mathrm{n}$ & 5 & 8,6 & 53 & 91,4 & \\
\hline \multirow{2}{*}{ Defectuosidad / Inamabilidad } & $\mathrm{Si}$ & $\mathrm{n}$ & 2 & 33,3 & 4 & 66,7 & \multirow{2}{*}{0,078} \\
\hline & No & $\mathrm{n}$ & 6 & 9,4 & 58 & 90,6 & \\
\hline \multirow{2}{*}{ Indeseabilidad Social } & $\mathrm{Si}$ & $\mathrm{n}$ & 1 & 16,7 & 5 & 83,3 & \multirow{2}{*}{0,673} \\
\hline & No & $\mathrm{n}$ & 7 & 10,9 & 57 & 89,1 & \\
\hline \multirow{2}{*}{ Incompetencia / Falla } & $\mathrm{Si}$ & $\mathrm{n}$ & 3 & 37,5 & 5 & 62,5 & \\
\hline & No & $\mathrm{n}$ & 5 & 8,1 & 57 & 91,9 & 0,014 \\
\hline Culng / Coctios & $\mathrm{Si}$ & $\mathrm{n}$ & 2 & 50,0 & 2 & 50,0 & \\
\hline cuipa / Castigo & No & $\mathrm{n}$ & 6 & 9,1 & 60 & 90,9 & 0,013 \\
\hline Pena / Vergüenza & $\mathrm{Si}$ & $\mathrm{n}$ & 1 & 25,0 & 3 & 75,0 & 0.380 \\
\hline & No & $\mathrm{n}$ & 7 & 10,6 & 59 & 89,4 & \\
\hline Limites Insuficientes / Engrandecimiento & $\mathrm{Si}$ & $\mathrm{n}$ & 4 & 30,8 & 9 & 69,2 & 0,015 \\
\hline Limites insuricientes / Engrandecimiento & No & $\mathrm{n}$ & 4 & 7,0 & 53 & 93,0 & 0,015 \\
\hline Autocontrol y Autodisciplina Insuficiente & $\mathrm{Si}$ & $\mathrm{n}$ & 1 & 11,1 & 8 & 88,9 & \\
\hline & No & $\mathrm{n}$ & 7 & 11,5 & 54 & 88,5 & 0,974 \\
\hline Estándares Inflexibles / Hipercrítica & $\mathrm{Si}$ & $\mathrm{n}$ & 3 & 30,0 & 7 & 70,0 & 0046 \\
\hline & No & $\mathrm{n}$ & 5 & 8,3 & 55 & 91,7 & 0,046 \\
\hline
\end{tabular}

Pte. $=$ presencia de la variable $; \mathrm{f}=$ frecuencia absoluta; $\mathrm{p}$ calculada por el chi cuadrado

\section{Fuente: Autores}

Teniendo como base los datos arrojados, se observa un alto nivel de asociación entre la variable de EMT's de sumisión/perdida de la individualidad, incompetencia/falla, culpa/castigo, límites insuficientes/ engrandecimiento y estándares inflexibles/hipercrítica, y la variable Ttno. de Ansiedad Generalizada 
Tabla 3. Tabla de contingencia EMT's \& Ttno de estrés postrauma

\begin{tabular}{|c|c|c|c|c|c|c|c|}
\hline \multirow{3}{*}{$\begin{array}{c}\text { ESQUEMAS } \\
\text { MALADAPTATIVOS TEMPRANOS }\end{array}$} & \multicolumn{7}{|c|}{ Ttno. de Estrés Postrauma } \\
\hline & \multirow[t]{2}{*}{ Pte. } & \multirow[t]{2}{*}{$\mathrm{f}$} & \multicolumn{2}{|c|}{$\mathrm{Si}$} & \multicolumn{2}{|c|}{ No } & \multirow[b]{2}{*}{$\mathrm{p}$} \\
\hline & & & $\mathrm{n}$ & $\%$ & $\mathrm{n}$ & $\%$ & \\
\hline \multirow{2}{*}{ Dependencia / Incompetencia } & $\mathrm{Si}$ & $\mathrm{n}$ & o & 0,0 & 12 & 100,0 & \multirow{2}{*}{0,291} \\
\hline & No & $\mathrm{n}$ & 5 & 8,6 & 53 & 91,4 & \\
\hline \multirow{2}{*}{ Vulnerabilidad al Daño y Enfermedad } & $\mathrm{Si}$ & $\mathrm{n}$ & o & 0,0 & 4 & 100,0 & \multirow{2}{*}{0,568} \\
\hline & No & $\mathrm{n}$ & 5 & 7,6 & 61 & 92,4 & \\
\hline \multirow{2}{*}{ Sumisión / Perdida Individualidad } & $\mathrm{Si}$ & $\mathrm{n}$ & o & 0,0 & 5 & 100,0 & \multirow[b]{2}{*}{0,520} \\
\hline & No & $\mathrm{n}$ & 5 & 7,7 & 60 & 92,3 & \\
\hline \multirow{2}{*}{$\begin{array}{l}\text { Temor a Perder el Control / Inhibición } \\
\text { Emocional }\end{array}$} & $\mathrm{Si}$ & $\mathrm{n}$ & o & 0,0 & 4 & 100,0 & \multirow{2}{*}{0,568} \\
\hline & No & $\mathrm{n}$ & 5 & 7,6 & 61 & 92,4 & \\
\hline \multirow{2}{*}{ Deprivación Emocional / Desamor } & $\mathrm{Si}$ & $\mathrm{n}$ & 1 & 9,1 & 10 & 90,9 & \multirow{2}{*}{0,785} \\
\hline & No & $\mathrm{n}$ & 4 & 6,8 & 55 & 93,2 & \\
\hline \multirow{2}{*}{ Abandono / Perdida } & $\mathrm{Si}$ & $\mathrm{n}$ & o & 0,0 & 10 & 100,0 & \multirow{2}{*}{0,343} \\
\hline & No & $\mathrm{n}$ & 5 & 8,3 & 55 & 91,7 & \\
\hline \multirow{2}{*}{ Desconfianza / Abuso } & $\mathrm{Si}$ & $\mathrm{n}$ & o & 0,0 & 13 & 100,0 & \multirow[b]{2}{*}{0,268} \\
\hline & No & $\mathrm{n}$ & 5 & 8,8 & 52 & 91,2 & \\
\hline \multirow{2}{*}{ Aislamiento Social / Alejamiento } & $\mathrm{Si}$ & $\mathrm{n}$ & $\mathrm{O}$ & 0,0 & 12 & 100,0 & \multirow[b]{2}{*}{0,291} \\
\hline & No & $\mathrm{n}$ & 5 & 8,6 & 53 & 91,4 & \\
\hline \multirow[b]{2}{*}{ Defectuosidad / Inamabilidad } & $\mathrm{Si}$ & $\mathrm{n}$ & 1 & 16,7 & 5 & 83,3 & \multirow[b]{2}{*}{0,343} \\
\hline & No & $\mathrm{n}$ & 4 & 6,3 & 60 & 93,8 & \\
\hline \multirow{2}{*}{ Indeseabilidad Social } & $\mathrm{Si}$ & $\mathrm{n}$ & O & 0,0 & 6 & 100,0 & \multirow[b]{2}{*}{0,477} \\
\hline & No & $\mathrm{n}$ & 5 & 7,8 & 59 & 92,2 & \\
\hline \multirow{2}{*}{ Incompetencia / Falla } & $\mathrm{Si}$ & $\mathrm{n}$ & o & 0,0 & 8 & 100,0 & 0405 \\
\hline & No & $\mathrm{n}$ & 5 & 8,1 & 57 & 91,9 & 0,405 \\
\hline Culne / Cestion & $\mathrm{Si}$ & $\mathrm{n}$ & o & 0,0 & 4 & 100,0 & \\
\hline Culpa / Castigo & No & $\mathrm{n}$ & 5 & 7,6 & 61 & 92,4 & 0,568 \\
\hline Pena / Vergüienza & $\mathrm{Si}$ & $\mathrm{n}$ & $\mathrm{O}$ & 0,0 & 4 & 100,0 & \\
\hline Pena / verguenza & No & $\mathrm{n}$ & 5 & 7,6 & 61 & 92,4 & 0,568 \\
\hline Limites Insuficientes / & $\mathrm{Si}$ & $\mathrm{n}$ & 3 & 23,1 & 10 & 76,9 & \\
\hline Engrandecimiento & No & $\mathrm{n}$ & 2 & 3,5 & 55 & 96,5 & 0,013 \\
\hline Autocontrol y Autodisciplina & $\mathrm{Si}$ & $\mathrm{n}$ & $\mathrm{O}$ & 0,0 & 9 & 100,0 & 272 \\
\hline Insuficiente & No & $\mathrm{n}$ & 5 & 8,2 & 56 & 91,8 & 0,373 \\
\hline Estándares Inflexibles / Hipercrítica & $\mathrm{Si}$ & $\mathrm{n}$ & 1 & 10,0 & 9 & 90,0 & 0,705 \\
\hline & No & $\mathrm{n}$ & 4 & 6,7 & 56 & 93,3 & \\
\hline
\end{tabular}

Pte. $=$ presencia de la variable; $\mathrm{f}=$ frecuencia absoluta; $\mathrm{p}$ calculada por el chi cuadrado

Fuente: Autores

Teniendo como base los datos arrojados, se observa un alto nivel de asociación entre la variable de EMT's de límites insuficientes/engrandecimiento, y la variable Ttno de Estrés Postrauma. 
Tabla 4. Tabla de contingencia EMT's \& Ttno de fobia específica

\begin{tabular}{|c|c|c|c|c|c|c|c|}
\hline \multirow{3}{*}{$\begin{array}{c}\text { ESQUEMAS } \\
\text { MALADAPTATIVOS TEMPRANOS }\end{array}$} & \multicolumn{7}{|c|}{ TtnodeFobia Específica } \\
\hline & \multirow{2}{*}{ Pte. } & \multirow[t]{2}{*}{$\mathrm{f}$} & \multicolumn{2}{|c|}{$\mathrm{Si}$} & \multicolumn{2}{|c|}{ No } & \multirow[b]{2}{*}{$\mathrm{p}$} \\
\hline & & & $\mathrm{n}$ & $\%$ & $\mathrm{~N}$ & $\%$ & \\
\hline \multirow[b]{2}{*}{ Dependencia / Incompetencia } & $\mathrm{Si}$ & $\mathrm{n}$ & 4 & 33,3 & 8 & 66,7 & \multirow[b]{2}{*}{0,507} \\
\hline & No & $\mathrm{n}$ & 14 & 24,1 & 44 & 75,9 & \\
\hline \multirow{2}{*}{ Vulnerabilidad al Daño y Enfermedad } & $\mathrm{Si}$ & $\mathrm{n}$ & 0 & 0,0 & 4 & 100,0 & \multirow[b]{2}{*}{0,226} \\
\hline & No & $\mathrm{n}$ & 18 & 27,3 & 48 & 72,7 & \\
\hline \multirow{2}{*}{ Sumisión / Perdida Individualidad } & $\mathrm{Si}$ & $\mathrm{n}$ & 2 & 40,0 & 3 & 60,0 & \multirow{2}{*}{0,448} \\
\hline & No & $\mathrm{n}$ & 16 & 24,6 & 49 & 75,4 & \\
\hline \multirow{2}{*}{$\begin{array}{l}\text { Temor a Perder el Control /Inhibición } \\
\text { Emocional }\end{array}$} & $\mathrm{Si}$ & $\mathrm{n}$ & o & 0,0 & 4 & 100,0 & \multirow[b]{2}{*}{0,226} \\
\hline & No & $\mathrm{n}$ & 18 & 27,3 & 48 & 72,7 & \\
\hline \multirow{2}{*}{ Deprivación Emocional / Desamor } & $\mathrm{Si}$ & $\mathrm{n}$ & o & 0,0 & 11 & 100,0 & \multirow{2}{*}{0,034} \\
\hline & No & $\mathrm{n}$ & 18 & 30,5 & 41 & 69,5 & \\
\hline \multirow{2}{*}{ Abandono / Perdida } & $\mathrm{Si}$ & $\mathrm{n}$ & 0 & 0,0 & 10 & 100,0 & \multirow{2}{*}{0,044} \\
\hline & No & $\mathrm{n}$ & 18 & 30,0 & 42 & 70,0 & \\
\hline \multirow{2}{*}{ Desconfianza / Abuso } & $\mathrm{Si}$ & $\mathrm{n}$ & 4 & 30,8 & 9 & 69,2 & \multirow{2}{*}{0,644} \\
\hline & No & $\mathrm{n}$ & 14 & 24,6 & 43 & 75,4 & \\
\hline \multirow{2}{*}{ Aislamiento Social / Alejamiento } & $\mathrm{Si}$ & $\mathrm{n}$ & 1 & 8,3 & 11 & 91,7 & \multirow{2}{*}{0,130} \\
\hline & No & $\mathrm{n}$ & 17 & 29,3 & 41 & 70,7 & \\
\hline \multirow{2}{*}{ Defectuosidad / Inamabilidad } & $\mathrm{Si}$ & $\mathrm{n}$ & o & 0,0 & 6 & 100,0 & \multirow{2}{*}{0,132} \\
\hline & & & 18 & & & 71,9 & \\
\hline \multirow[t]{2}{*}{ Indeseabilidad Social } & $\mathrm{Si}$ & $\mathrm{n}$ & $\mathrm{O}$ & 0,0 & 6 & 100,0 & \multirow{2}{*}{0,132} \\
\hline & & & 18 & & & 71,9 & \\
\hline \multirow{2}{*}{ Incompetencia / Falla } & $\mathrm{Si}$ & $\mathrm{n}$ & 1 & 12,5 & 7 & 87,5 & 0,364 \\
\hline & No & $\mathrm{n}$ & 17 & 27,4 & 45 & 72,6 & \\
\hline Culpa / Castigo & $\mathrm{Si}$ & $\mathrm{n}$ & 1 & 25,0 & 3 & 75,0 & \\
\hline & No & $\mathrm{n}$ & 17 & 25,8 & 49 & 74,2 & 0,973 \\
\hline Pena / Vergüenza & $\mathrm{Si}$ & $\mathrm{n}$ & 0 & 0,0 & 4 & 100,0 & 0,226 \\
\hline Limites Insuficientes / & $\mathrm{Si}$ & $n$ & 4 & 308 & 9 & 69,2 & \\
\hline Engrandecimiento & No & $\mathrm{n}$ & 14 & 24,6 & 43 & 75,4 & 0,644 \\
\hline Autocontrol y Autodisciplina & $\mathrm{Si}$ & $\mathrm{n}$ & 4 & 44,4 & 5 & 55,6 & 460 \\
\hline Insuficiente & No & $\mathrm{n}$ & 14 & 23,0 & 47 & 77,0 & 0,108 \\
\hline Estándares Inflexibles / & $\mathrm{Si}$ & $\mathrm{n}$ & o & 0,0 & 10 & 100,0 & 0,044 \\
\hline Hipercrítica & No & $\mathrm{n}$ & 18 & 30,0 & 42 & 70,0 & \\
\hline
\end{tabular}

Pte. $=$ presencia de la variable; $\mathrm{f}=$ frecuencia absoluta; $\mathrm{p}$ calculada por el chi cuadrado

\section{Fuente: Autores}

Teniendo como base los datos arrojados, se observa un alto nivel de asociación entre la variable de EMT's de deprivación emocional/ desamor, abandono/perdida, estándares inflexibles/hipercrítica, y la variable Ttno. de fobia específica. 
Tabla 5. Tabla de contingencia EMT's \& Ttno. de pánico.

\begin{tabular}{|c|c|c|c|c|c|c|c|}
\hline \multirow{3}{*}{$\begin{array}{c}\text { ESQUEMAS } \\
\text { MALADAPTATIVOS TEMPRANOS }\end{array}$} & \multicolumn{7}{|c|}{ Ttno. de Pánico } \\
\hline & \multirow[t]{2}{*}{ Pte. } & \multirow[t]{2}{*}{$\mathrm{f}$} & \multicolumn{2}{|c|}{$\mathrm{Si}$} & \multicolumn{3}{|c|}{ No } \\
\hline & & & $\mathrm{n}$ & $\%$ & $\mathrm{n}$ & $\%$ & $\mathrm{p}$ \\
\hline \multirow{2}{*}{ Dependencia / Incompetencia } & $\mathrm{Si}$ & $\mathrm{n}$ & o & 0,0 & 12 & 100,0 & \multirow[b]{2}{*}{0,349} \\
\hline & No & $\mathrm{n}$ & 4 & 6,9 & 54 & 93,1 & \\
\hline \multirow{2}{*}{ Vulnerabilidad al Daño y Enfermedad } & $\mathrm{Si}$ & $\mathrm{n}$ & 2 & 50,0 & 2 & 50,0 & \multirow{2}{*}{0,000} \\
\hline & No & $\mathrm{n}$ & 2 & 3,0 & 64 & 97,0 & \\
\hline \multirow{2}{*}{ Sumisión / Perdida Individualidad } & $\mathrm{Si}$ & $\mathrm{n}$ & o & 0,0 & 5 & 100,0 & \multirow{2}{*}{0,568} \\
\hline & No & $\mathrm{n}$ & 4 & 6,2 & 61 & 93,8 & \\
\hline \multirow{2}{*}{$\begin{array}{l}\text { Temor a Perder el Control / } \\
\text { Inhibición Emocional }\end{array}$} & $\mathrm{Si}$ & $\mathrm{n}$ & 2 & 50,0 & 2 & 50,0 & \multirow{2}{*}{0,000} \\
\hline & No & $\mathrm{n}$ & 2 & 3,0 & 64 & 97,0 & \\
\hline \multirow{2}{*}{ Deprivación Emocional / Desamor } & $\mathrm{Si}$ & $\mathrm{n}$ & 2 & 18,2 & 9 & 81,8 & \multirow{2}{*}{0,052} \\
\hline & No & $\mathrm{n}$ & 2 & 3,4 & 57 & 96,6 & \\
\hline Abandono / Perdida & $\mathrm{Si}$ & $\mathrm{n}$ & 1 & 10,0 & 9 & 90,0 & 0,528 \\
\hline \multirow[b]{2}{*}{ Desconfianza / Abuso } & $\mathrm{Si}$ & $\mathrm{n}$ & $\frac{3}{2}$ & & 11 & $\frac{95,0}{84,6}$ & \multirow[b]{2}{*}{0,096} \\
\hline & No & $\mathrm{n}$ & $\begin{array}{l}2 \\
2 \\
\end{array}$ & $\begin{array}{r}15,4 \\
3,5 \\
\end{array}$ & 55 & $\begin{array}{r}84,0 \\
96,5 \\
\end{array}$ & \\
\hline \multirow[t]{2}{*}{ Aislamiento Social /Alejamiento } & $\mathrm{Si}$ & $\mathrm{n}$ & o & 0,0 & 12 & 100,0 & \multirow{2}{*}{0,349} \\
\hline & No & $\mathrm{n}$ & 4 & 6,9 & 54 & & \\
\hline \multirow[t]{2}{*}{ Defectuosidad / Inamabilidad } & $\mathrm{Si}$ & $\mathrm{n}$ & 1 & 16,7 & 5 & 83,3 & \multirow{2}{*}{0,227} \\
\hline & No & $\mathrm{n}$ & 3 & 4,7 & 61 & 95,3 & \\
\hline \multirow[t]{2}{*}{ Indeseabilidad Social } & $\mathrm{Si}$ & $\mathrm{n}$ & o & 0,0 & 6 & 100,0 & \multirow{2}{*}{0,528} \\
\hline & No & $\mathrm{n}$ & 4 & 6,3 & 60 & 93,8 & \\
\hline \multirow{2}{*}{ Incompetencia / Falla } & $\mathrm{Si}$ & $\mathrm{n}$ & $\mathrm{o}$ & 0,0 & 8 & 100,0 & \multirow{2}{*}{0,459} \\
\hline & No & $\mathrm{n}$ & 4 & 6,5 & 58 & 93,5 & \\
\hline Culpa / Castigo & $\mathrm{Si}$ & $\mathrm{n}$ & 0 & 0,0 & 4 & 100,0 & \\
\hline & No & $\mathrm{n}$ & 4 & 6,1 & 62 & 93,9 & 0,612 \\
\hline Pena / Vergüenza & $\mathrm{Si}$ & $\mathrm{n}$ & 1 & 25,0 & 3 & 75,0 & 0.087 \\
\hline & No & $\mathrm{n}$ & 3 & 4,5 & 63 & 95,5 & 0,087 \\
\hline Limites Insuficientes / & $\mathrm{Si}$ & $\mathrm{n}$ & 2 & 15,4 & 11 & 84,6 & \\
\hline Engrandecimiento & No & $\mathrm{n}$ & 2 & 3,5 & 55 & 96,5 & 0,096 \\
\hline Autocontrol y Autodisciplina & $\mathrm{Si}$ & $\mathrm{n}$ & $\mathrm{o}$ & 0,0 & 9 & 100,0 & \\
\hline Insuficiente & No & $\mathrm{n}$ & 4 & 6,6 & 57 & 93,4 & 0,429 \\
\hline Estándares Inflexibles / Hipercrítica & $\mathrm{Si}$ & $\mathrm{n}$ & 2 & 20,0 & 8 & 80,0 & 0,036 \\
\hline & No & $\mathrm{n}$ & 2 & 3,3 & 58 & 96,7 & \\
\hline
\end{tabular}

Pte. $=$ presencia de la variable; $\mathrm{f}=$ frecuencia absoluta; $\mathrm{p}$ calculada por el chi cuadrado

Fuente: Autores

Teniendo como base los datos arrojados, se observa un alto nivel de asociación entre la variable de EMT's de vulnerabilidad al daño y a la enfermedad, temor a perder el control/inhibición emocional, estándares inflexibles/hipercrítica, y la variable Ttno. de pánico. 


\section{Discusión de resultados}

El siguiente análisis se realiza a partir de la teoría de los esquemas maladaptativos tempranos de Jeffrey Young tomando como base la información obtenida a partir de los resultados de las pruebas y su posterior procesamiento a través del software SPSS 22.

De acuerdo con los resultados de la investigación, se observa relación entre algunos EMT's y los trastornos de ansiedad tenidos en cuenta en el estudio. Se rechaza entonces la hipótesis nula $\left(\mathrm{H}_{\mathrm{o}}\right)$, y se confirma la hipótesis alternativa $\left(\mathrm{H}_{1}\right)$, la que indica que si existen EMT's relacionados con la aparición de los trastornos de ansiedad.

Específicamente con respecto a la relación entre los esquemas maladaptativos tempranos y el trastornos de ansiedad generalizada y teniendo como base los datos arrojados, se observa un alto nivel de asociación entre la variable de EMT's de sumisión/perdida de la individualidad, incompetencia/falla, culpa/castigo, límites insuficientes/ engrandecimiento y estándares inflexibles/hipercrítica, y la variable Ttno. de ansiedad generalizada.

El EMT's de sumisión/perdida individualidad hace referencia a la entrega excesiva del control sobre nuestras propias decisiones, preferencias y expresión emocional en las manos de los demás; generalmente para evitar la ira, las represalias o el abandono. Conlleva la percepción de que nuestros propios sentimientos y deseos no son válidos o importantes para los demás, lo cual implica su sacrificio para satisfacer los de los otros. Con frecuencia conduce a dificultades para establecer metas y dirección en la vida, así como a fallas en el reconocimiento de mis propias necesidades, y termina generando la ira hacia el subyugador (Young, 1993).

Este esquema pertenece al área de la autonomía, manifestándose en el niño a través de una sensación de minusvalía de sus propias necesidades y la incapacidad para expresar sus sentimientos y pedir ayuda, lo cual lo lleva a sentirse vulnerable y pasivo frente a un mundo al cual percibe como fuera de su control. 
La sensación de control sobre el ambiente es importante, porque facilita que el ser humano se enfrentarse a los potenciales peligros del ambiente que le rodea manteniendo expectativas de éxito frente a dicha tarea, pero en el caso de este esquema maladaptativo el niño suele permanecer pasivo, callado y ensimismado perdiendo la oportunidad de pedir ayuda o enfrentarse a los peligros al no ser muy consciente de sus propias necesidades y fortalezas. Este esquema operaria por el proceso de mantenimiento tipo rendición en relación al trastorno de ansiedad generalizada.

El EMT's de incompetencia/falla, hace referencia la creencia de que uno no se puede desempeñar competentemente en áreas de logro (académica, profesional, social o personalmente). Que es incapaz de asumir las responsabilidades cotidianas con uno mismo y con los demás, y que no le es posible tomar sus propias decisiones. (Young, 1993).

Cuando se presenta una visión de sí mismo caracterizada por el fracaso y una escaza habilidad para enfrentar el medio, dicha autopercepción implicaría sentirse incapaz para afrontar las amenazas externas de manera eficiente. Para el niño con constantes sentimientos de fracaso es sumamente difícil enfrentarse a las diferentes situaciones de la vida a las cuales percibiría como demasiado complejas y atemorizantes, si la experiencia del niño implica una constante vivencia de miedo, este podría derivar finalmente en un cuadro ansioso. Cuando se tiene una vivencia de incapacidad para enfrentar el mundo, la percepción de este suele ser bastante amenazante. Este esquema operaria por el proceso de mantenimiento tipo rendición, como una variable de predisposición psicológica para el desarrollo del trastorno en cuestión.

El EMT's de culpa/castigo hace referencia a la creencia de que uno es moral o éticamente malo o irresponsable y por lo tanto merecedor o digno de duras críticas o castigos (Young, 1993).

Según la teoría de los esquemas maladaptativos los niños con este esquema, suelen presentar la sensación de haber actuado mal o de haber hecho o pensado algo que para ellos es horrible. La precepción ne- 
gativa de sí mismo con respecto a la moralidad del propio ser incurre en intensos estados de angustia causados por la culpa. Dichos estados de angustia y la idea de que no se merece el apoyo de sus cuidadores puede convertirse en una variable de predisposición psicológica para la generación de cuadros ansiosos significativos. El proceso de mantenimiento que opera en el esquema es por rendición, facilitando en el sujeto la idea de que eventos adversos y grandes amenazas podrían ocurrir como consecuencia de su maldad moral a modo de castigo.

El EMT's de límites insuficientes/engrandecimiento hace referencia a la idea de que uno debería tener lo que quiera en el mismo momento en que lo desee, o que podría decir o hacer lo que le provoque en cualquier momento, sin importar ninguna razón. También implica la creencia de que se es superior a otras personas, que se poseen derechos y privilegios especiales o que no debe regirse por las reglas de reciprocidad que guían las interacciones sociales normales (Young, 1993)

La presencia de este esquema implica en el niño el uso inadecuado de estrategias de autocontrol o incluso la carencia total de las mismas, que, si existieran, podrían en un momento dado protegerlo de inminentes peligros. La vivencia de autocontrol también es importante para la consolidación de la visión de un mundo controlable y seguro. El proceso de mantenimiento que opera en el esquema es por rendición, de esta manera la incapacidad del niño para controlar sus impulsos y emociones se refleja en la percepción de un ambiente igualmente descontrolado y por ende amenazante, además de inadecuadas estrategias para el procesamiento emocional.

El EMT's de estándares inflexibles/hipercrítica hace referencia al implacable esfuerzo por lograr o cumplir expectativas que solo podrían alcanzarse a expensas de la felicidad, el placer, la salud, el sentido de compañía o relaciones interpersonales satisfactorias (Young, 1993).

Puede llevar al niño a ser poco consciente de sus necesidades emocionales y a manejar un nivel de auto exigencia poco realista, lo cual suele relacionarse con estados de angustia. El proceso de manteni- 
miento que opera en el esquema es por rendición, generando en el infante un sentimiento de permanente presión sobre el logro de expectativas que nunca logra alcanzar, con la consecuente incapacidad de vivenciar la satisfacción, llevándolo a la frustración personal. Este esquema actuaría entonces como una característica de vulnerabilidad psicológica para el desarrollo del trastorno de ansiedad.

Con respecto a la relación entre los esquemas maladaptativos tempranos y el Ttno. de estrés postrauma, se observa un alto nivel de asociación entre la variable de EMT's de límites insuficientes/engrandecimiento, y la variable Ttno de estrés postrauma.

El EMT's de límites insuficientes/engrandecimiento hace referencia a la idea de que uno debería tener lo que quiera en el mismo momento en que lo desee, o que podría decir o hacer lo que le provoque en cualquier momento, sin importar ninguna razón. También implica la creencia de que se es superior a otras personas, que se poseen derechos y privilegios especiales o que no debe regirse por las reglas de reciprocidad que guían las interacciones sociales normales (Young, 1993)

Aparece nuevamente la noción de no control sobre el ambiente como consecuencia de la propia incapacidad para controlarse y contenerse. El proceso de mantenimiento que opera en el esquema es por rendición, lo que interfiere con la capacidad del niño para controlar la emoción del miedo e interfiriendo sobre el adecuado procesamiento del evento estresante a través de dicha emoción primaria (miedo) cumpliendo con su función adaptativa, y transformándose en emoción secundaria (ansiedad) la cual se manifestaría por cuadros de angustia y malestar asociados a los recuerdos e imágenes del evento estresor.

Con respecto a la relación entre los esquemas maladaptativos tempranos y el Ttno. de fobia específica, se observa un alto nivel de asociación entre la variable de EMT's de deprivación emocional/desamor, abandono/perdida, estándares inflexibles/hipercrítica, y la variable Ttno. de fobia específica. 
EMT's de deprivación emocional/desamor hace referencia a la creencia de que las propias necesidades de afecto, empatía y cuidado nunca serán satisfechas por los demás de forma adecuada. También involucra el sentimiento de no ser comprendido, escuchado, valorado o apoyado de forma adecuada por los demás (Young, 1993).

Un ambiente que no propicia un sentimiento de seguridad emocional en el niño, a la vez puede favorecer el desarrollo de estados emocionales ansiosos los cuales se agravarían frente a la presencia de algún evento altamente estresante para él. El proceso de mantenimiento que opera en el esquema es por rendición.

El EMT's de abandono/perdida hace referencia a el temor de que uno, de manera inminente por cualquier razón, sufrirá la pérdida de personas significativas en su vida y luego estará emocionalmente aislado por siempre en total desconexión (Young, 1993).

La vivencia de abandono se ha correlacionado directamente en la teoría de los esquemas maladaptativos tempranos de Young y la teoría del apego de John Bowlby. Para este último, el niño frente a la posibilidad de la pérdida de su figura de apego, manifiesta tres etapas que son angustia, depresión e ira y, finalmente, indiferencia (Young, \& Klosko, 1993).

Los niños cuyo esquema de abandono sea activado pueden llegar a manifestar en algunos casos el ciclo completo pasando por las tres fases, o solo manifestando una de ellas (Young, \& Klosko1994).

En el caso de la primera fase del abandono se presentarían conductas y síntomas de ansiedad que podrían llegar a configurarse y consolidarse con el tiempo y la repetición en un cuadro fóbico frente a la separación o alejamiento de la figura de apego, en donde el aversivo sería precisamente la perdida de dicha figura. El proceso de mantenimiento que opera en el esquema es por rendición.

EMT's de estándares inflexibles/hipercrítica el cual hace referencia al implacable esfuerzo por lograr o cumplir expectativas que solo podrían alcanzarse a expensas de la felicidad, el placer, la salud, el sentido de compañía o relaciones interpersonales satisfactorias (Young, 1993). 
Nuevamente aparece este esquema asociado a un cuadro de ansiedad. Como se había afirmado anteriormente los niños con este esquema suelen ser poco conscientes de sus necesidades emocionales y a manejar un nivel de auto exigencia excesiva que en ocasiones puede llegar a ser patológico. Este esquema parece actuar como una característica de vulnerabilidad psicológica concomitante para el desarrollo de diferentes trastornos de ansiedad. El proceso de mantenimiento que opera en el esquema es por rendición.

Con respecto a la relación entre los esquemas maladaptativos tempranos y el Ttno. de pánico, se observa un alto nivel de asociación entre la variable de EMT's de vulnerabilidad al daño y a la enfermedad, temor a perder el control/inhibición emocional, estándares inflexibles / hipercrítica, y la variable Ttno. de Pánico.

EMT's de temor a perder el control/inhibición emocional hace referencia al temor a que uno, de manera inminente e involuntaria, perderá el control de su propia conducta, sus impulsos, sus emociones, su cuerpo o su mente si se permite sentir y expresar sus emociones (Young, 1993).

El temor a perder el control y la inhibición emocional no favorecen la expresión de las emociones en el sujeto, generando estados de tensión interna que con el tiempo pueden desencadenar en ataques de pánico por la saturación del sistema neurocognitivo y la incapacidad de el mismo para continuar manteniendo el control interno una vez se han agotado los recursos para llevar a cabo esta tarea.

Igualmente el inadecuado procesamiento de las emociones al estar bloqueadas y no ser expresadas, suelen derivar en emociones de tipo secundario, en este caso particular la emoción secundaria de ansiedad (Zaraï, 1996), facilitando el desarrollo de patologías asociadas a la misma. El proceso de mantenimiento que opera en el esquema es por evitación.

EMT's de vulnerabilidad al daño y a la enfermedad hace referencia al exagerado temor a que la calamidad o el desastre esté a punto de golpearnos en cualquier momento, a nivel natural, criminal, salud, y que uno es incapaz de protegerse a sí mismo (Young, 1993). 
La visión que suelen tener los niños sobre sí mismos que poseen este esquema gira en torno a ser seres vulnerables a los desastres, las enfermedades y un sinnúmero de daños potenciales que pondrían en riesgo su integridad física sintiéndose impotentes para poder protegerse. El proceso de mantenimiento que opera en el esquema es por rendición generando un estado de predisposición psicológica para el desarrollo del trastorno.

EMT's de estándares inflexibles/hipercrítica el cual hace referencia al implacable esfuerzo por lograr o cumplir expectativas que solo podrían alcanzarse a expensas de la felicidad, el placer, la salud, el sentido de compañía o relaciones interpersonales satisfactorias (Young, 1993).

De nuevo este esquema aparece en el marco de los trastornos de ansiedad de la población estudiada. Este esquema facilita el desarrollo patológico de estados internos de tensión emocional y angustia por la presión percibida por el niño que lo obliga a alcanzar metas y estándares irracionales que, por supuesto nunca logra alcanzar, tensión emocional que es controlada de manera excesiva gracias a la presencia del esquema de temor a perder el control / inhibición emocional. El proceso de mantenimiento que opera en el esquema es por rendición, convirtiéndolo nuevamente en una característica de vulnerabilidad psicológica para el desarrollo de este u otro trastorno de ansiedad.

\section{Conclusiones}

Con respecto al trastorno de ansiedad generalizada, puede observarse la conjunción como vulnerabilidad psicológica de características en el niños tales como incapacidad para reconocer las propias necesidades y fortalezas, incapacidad para expresar dichas necesidades de manera clara y segura, invalidación de los propios deseos, dificultades para establecer metas claras en la vida, una marcada sensación de no control no predicción frente al ambiente, dificultad en la toma de decisiones, auto estima caracterizado por sentimientos de fracaso e inhabilidad y eventualmente profundos sentimientos de culpa relacionado con una idea de sí mismo de ser malo y merecedor de duros castigos. 
Igualmente una marcada incapacidad para el autocontrol y estar bajo un alto nivel de presión por parte del medio para alcanzar metas irracionales que solo lo dejaran en la frustración. Las áreas de desarrollo implicadas en el trastorno son: el área de la autonomía (un esquema) y el área de autovaloración (dos esquemas), el área de límites razonables (un esquema), y el área de estándares (un esquema).

En relación al trastorno de estrés postrauma se observa específicamente la incapacidad del niño para auto controlar y auto regular sus emociones, predisposición psicológica que en combinación con la aparición de un evento altamente estresante podría facilitar el desarrollo del trastorno. El área de desarrollo implicada en el trastorno es el área de límites razonables (un esquema).

En el trastorno de fobia específica parecen operar aspectos como la insatisfacción de las necesidades afectivas, de empatía y cuidado, nuevamente estar bajo un alto nivel de presión por parte del medio para alcanzar metas irracionales y de manera muy importante la incapacidad por parte del niño de tolerar la separación o ausencia de la figura de apego por tiempos incluso insignificantes. Las áreas de desarrollo implicadas en el trastorno son: el área de las relaciones interpersonales (dos esquemas) y el área de estándares (un esquema).

El trastorno de pánico se ve potenciado por la presencia de características psicológicas tales como el bloqueo emocional, la incapacidad e expresar las emociones, los sentimientos de vulnerabilidad y fragilidad frente a las posibles amenazas externas; la presión externa para alcanzar metas irracionales a expensas de la salud y el placer. Las áreas de desarrollo implicadas en el trastorno son: el área de la autonomía (dos esquemas) y el área de estándares (un esquema).

Se observa que el EMT's de estándares inflexibles/hipercrítica está implicado en los trastornos de ansiedad generalizada, fobia específica y pánico, convirtiéndolo en un aspecto a tener en cuenta como una variable potencialmente dañina y coadyuvante en el desarrollo de diferentes trastornos de ansiedad. 
Otro esquema que debe ser tenido en cuenta al momento de la evaluación de los trastornos de ansiedad en los niños, pues aparece implicado en Ttno. de ansiedad generalizada y el de estrés postrauma, es el EMT's de límites insuficientes / engrandecimiento, interfiriendo en el niño en el saludable desarrollo de estrategias de autocontrol y autorregulación emocional que pudieran facilitar el procesamiento de las emociones, específicamente en este caso el de la emoción primaria del miedo.

Las áreas de desarrollo mayormente implicadas en relación con los trastornos estudiados y a las cuales se les debe prestar importante atención al momento de la evaluación psicológica, son el área de la autonomía y el área de los estándares. Igualmente el fortalecimiento de dichas áreas podría prevenir la aparición de dichos trastornos.

Los procesos de mantenimiento de los EMT's encontrados en esta investigación se dan por mayoría por el tipo rendición, dejando a los niños vulnerables y sin estrategias para el afrontamiento de situaciones generadoras de ansiedad.

En conclusión, una visión de sí mismo en el niño caracterizada por el desconocimiento de las propias necesidades y fortalezas; la incapacidad para expresar lo que necesita; el sentirse vulnerable frente a un mundo que considera amenazante; la escasa capacidad de autocontrol y autorregulación; y una presión excesiva por parte del medio, se convierten en importantes variables de predisposición psicológica para el desarrollo de sintomatología ansiosa, e incluso trastornos de ansiedad. 


\section{Referencias}

American Psychological Association. (2014). DSM-V. Recuperado de http://www.medicapanamericana.com/Libros/Libro/4949/DSM5-Manual-Diagnostico-y-Estadistico-de-los-Trastornos-Mentales.html.

Bowlby, J. (1993). El vínculo afectivo. Barcelona. Paidós.

Caro, I. (1997). Manual de psicoterapias cognitivas. Buenos Aires. Ed. Paidós.

Méndez, F. X., Ingles, C. J., Hidalgo, M. D., García, J. M., Quiles, M. J. (2003) Los miedos en la infancia y la adolescencia: un estudio descriptivo. Revista Electrónica de Motivación y Emoción; 13 (6). Recuperado de http://reme.uji.es/ articulos/amxndf4650710102/texto.html.

Organización Mundial de la Salud. (2016). ICD-1O. Recuperado de http://apps.who. int/classifications/icd10/browse/2016/en.

SEAS (s.f.). Sociedad española para el estudio de la ansiedad y el estrés. Recuperado de http://pendientedemigracion.ucm.es/info/seas/ta/introduc.htm.

Young, J. \& Klosko, J. (1993). Reinventa tu vida. Barcelona: Paidós.

Young, J. (1993). Terapia centrada en esquemas para trastornos de personalidad. Barcelona: Paidós.

Young, J. \& Klosko, J. (1994). Reinventing your life: The breakthrough program to end negative behaivor and feel great again. USA: Plume.

Young, J. (1999). Cognitive therapy for personality disorders: a schema-focused approach. (practitioner's resource series). USA: Professional Resource Exchange In.

Zaraï, R. (1996). Las emociones que curan. Bogotá: Ed. Nueva Era. 